\title{
Plasmonic nanoparticle enhanced photocurrent in GaN/InGaN/GaN quantum well solar cells
}

\author{
Imogen M. Pryce, ${ }^{1, a)}$ Daniel D. Koleske, ${ }^{2}$ Arthur J. Fischer, ${ }^{2}$ and Harry A. Atwater ${ }^{1,3}$ \\ ${ }^{1}$ Thomas J Watson Laboratories of Applied Physics, California Institute of Technology, Pasadena, \\ California 91125, USA \\ ${ }^{2}$ Semiconductor Materials and Device Sciences, Sandia National Laboratories, Albuquerque, \\ New Mexico 87185, USA \\ ${ }^{3}$ Kavli Nanoscience Institute, California Institute of Technology, Pasadena, \\ California 91125, USA
}

(Received 26 February 2010; accepted 11 March 2010; published online 12 April 2010; publisher error corrected 20 April 2010)

\begin{abstract}
We demonstrate enhanced external quantum efficiency and current-voltage characteristics due to scattering by $100 \mathrm{~nm}$ silver nanoparticles in a single $2.5 \mathrm{~nm}$ thick InGaN quantum well photovoltaic device. Nanoparticle arrays were fabricated on the surface of the device using an anodic alumina template masking process. The Ag nanoparticles increase light scattering, light trapping, and carrier collection in the III-N semiconductor layers leading to enhancement of the external quantum efficiency by up to 54\%. Additionally, the short-circuit current in cells with $200 \mathrm{~nm} \mathrm{p}$-GaN emitter regions is increased by $6 \%$ under AM 1.5 illumination. AFORS-Het simulation software results were used to predict cell performance and optimize emitter layer thickness. () 2010 American Institute of Physics. [doi:10.1063/1.3377900]
\end{abstract}

Plasmonic nanoparticle scattering offers a unique way to circumvent the inherent trade-off between absorption and carrier collection in the design of solar cells. Optically thick cells can absorb all nonreflected incident light but incomplete carrier collection can limit cell internal quantum efficiency. The thickness reduction of high efficiency, low cost, thin film solar cells is limited by the absorption properties of the active layer. Increasing cell efficiency and reducing the material thickness both motivate investigating ways of increasing the light absorption in photovoltaic materials. Plasmonic nanostructures have been studied on both optically thick and thin semiconductor films. ${ }^{1-8}$ Conventional solar cells often incorporate surface texturing to increase the optical path length of light within the cell but these structures are too large to be used in thin film cells. ${ }^{9}$ Metal nanoparticles can serve a similar purpose in very thin cells, where surface texturing is not possible, due to their large scattering crosssections and enhanced local fields. ${ }^{10}$ For thicker cells, the increase in optical path length translates to an increase in effective optical thickness of the active region. In the case of thin film cells, there is an additional benefit: the metal nanostructures can couple incident light into guided modes that propagate through the active region, thereby increasing absorption and photocurrent. Here, we demonstrate that nanoparticle light scattering can be tailored to enhance absorption in optically thin InGaN single quantum well $(\mathrm{QW})$ based photovoltaic devices.

Recent breakthroughs in the ability to grow higher quality InGaN alloys have made them materials of particular interest for photovoltaic applications. The InGaN material system can be compositionally tuned across the visible portion of the solar spectrum, maintaining a direct band gap throughout the composition gradient. ${ }^{11}$ InGaN materials also exhibit superior radiation resistance making them suitable for spacebased photovoltaics. ${ }^{11}$ Several preliminary InGaN-based de-

${ }^{a)}$ Electronic mail: imogen@caltech.edu. vice designs have been demonstrated; ${ }^{12-14}$ however, these devices have been limited by film quality due to strain and phase separation. QW-based architectures are relatively tolerant to lattice mismatch and have been used extensively in light emitting diodes (LEDs) and laser diodes at wavelengths through the visible spectrum. In this paper we show that the absorption in a QW-based device can be enhanced, enabling the development of photovoltaic devices from material previously suitable only for LEDs.

The $\mathrm{GaN} / \mathrm{InGaN} / \mathrm{GaN}$ structures employed here were initially designed as LEDs but also serve here as prototypical extremely thin photovoltaic devices for probing enhanced quantum well absorption. Figure 1(a) shows a schematic of the QW samples grown via metalorganic chemical vapor deposition. A $2-3 \mu \mathrm{m} \mathrm{n}-\mathrm{GaN}$ buffer layer (n-doped [Si]

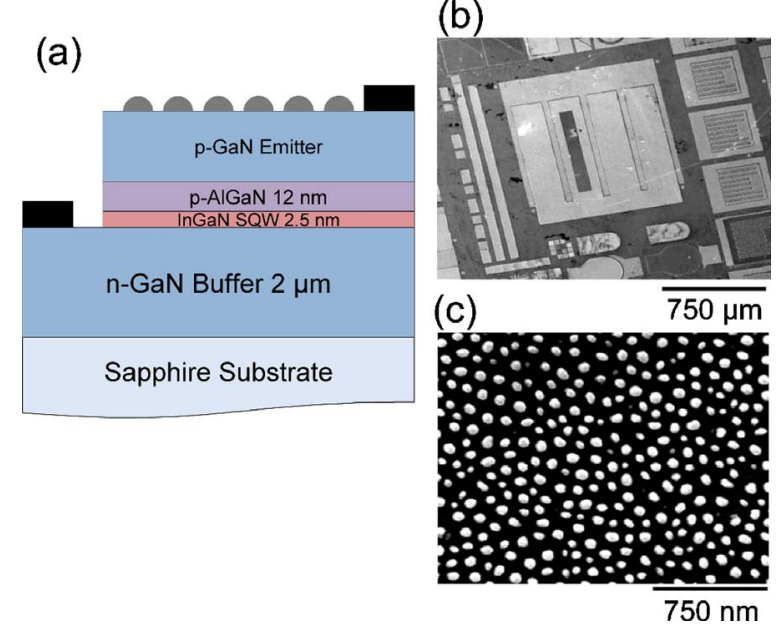

FIG. 1. (Color online) (a) A schematic of the device structure with Ag nanoparticles; (b) an SEM image of the actual device with a window etched into the top contact for nanoparticle deposition; (c) Ag nanoparticles spaced $200 \mathrm{~nm}$ apart, with $100 \mathrm{~nm}$ diameter and height, deposited onto the QW cell via thermal evaporation through an anodic aluminum oxide template that is lifted off. 


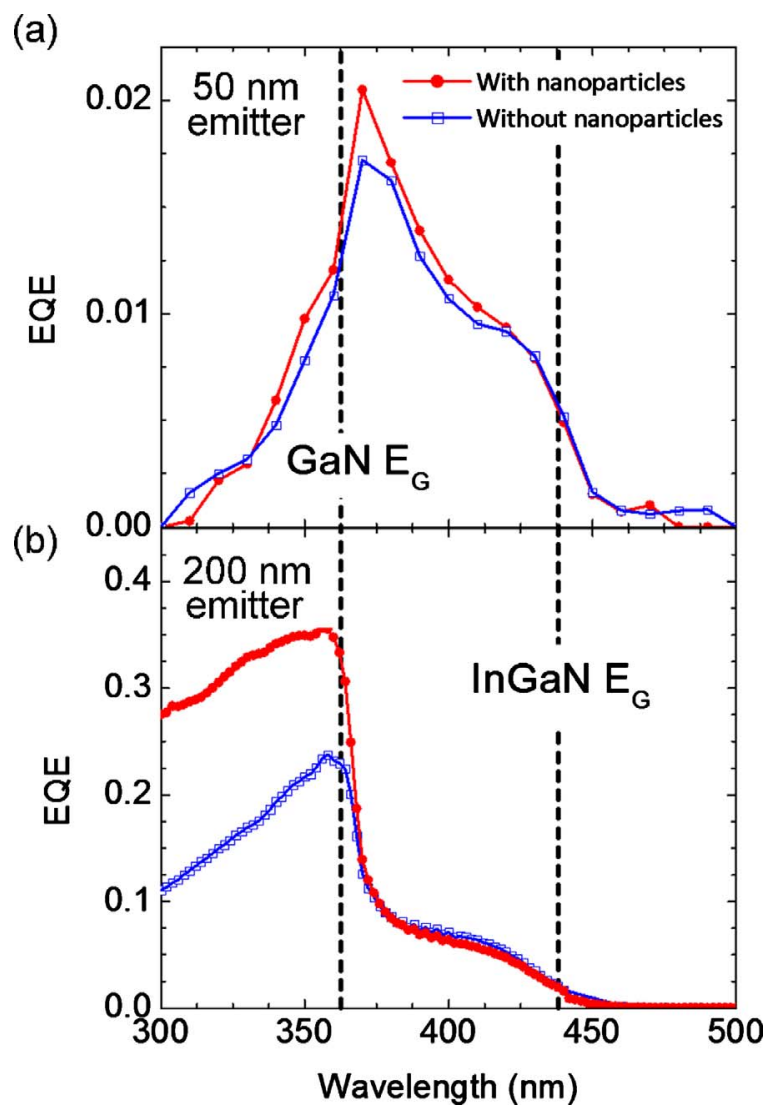

FIG. 2. (Color online) (a) EQE curves of a QW cell with a $50 \mathrm{~nm}$ p-GaN emitter layer before and after nanoparticle deposition; (b) EQE curves of a QW cell with a $200 \mathrm{~nm}$ p-GaN emitter layer before and after nanoparticle deposition.

$=2-3 \times 10^{18} \mathrm{~cm}^{-3}$ ) is first deposited on a sapphire substrate followed by a $2.5 \mathrm{~nm}$ InGaN QW with a band gap of $2.8 \mathrm{eV}$. A $12 \mathrm{~nm}$ p-AlGaN electron blocking layer is deposited on top of the QW, followed by a p-GaN emitter layer (p-doped $\left.[\mathrm{Mg}]=3-5 \times 10^{17} \mathrm{~cm}^{-3}\right)$. A $7.5 \times 10^{-4} \mathrm{~cm}^{2}$ window is etched into each device via $\mathrm{Ar}^{+}$ion milling of the top p-contact to facilitate nanoparticle deposition [Fig. 1(b)]. The p-GaN deposited thickness is $200 \mathrm{~nm}$, however, this is varied by changing the duration of the etch. Two samples of different emitter layer thickness, 50 and $200 \mathrm{~nm}$, were studied.

We deposit Ag nanoparticles using a porous anodic aluminum oxide masking technique. ${ }^{8}$ This has the advantage of being inexpensive and provides control over size, height, and spacing of the nanoparticles. $\mathrm{Al}$ sheets $(4 \mathrm{~N})$ are anodized in a two-step process at $80 \mathrm{~V}$ in a mixture of $0.3 \mathrm{M}$ oxalic acid and $0.3 \mathrm{M}$ malonic acid to yield an interpore spacing of 200 $\mathrm{nm}$. The $\mathrm{Al}$ is removed using an iodine-methanol solution leaving a porous aluminum oxide sheet. The mask template is etched in 5\% dilute phosphoric acid for $65 \mathrm{~min}$ to yield pore diameters of $100 \mathrm{~nm}$. The template is then placed in direct contact with the device window and used as a mask for thermal evaporation of Ag. The resulting nanoparticles are approximately $100 \mathrm{~nm}$ in diameter and height, with $200 \mathrm{~nm}$ spacing. Figure 1(c) shows a scanning electron microscopy (SEM) image of a representative nanoparticle array. The irregularity in periodicity is characteristic of this type of deposition.

External quantum efficiency (EQE) measurements are used to quantify how the absorption varies spectrally for devices coated with nanoparticle arrays. Figure 2 shows spec-

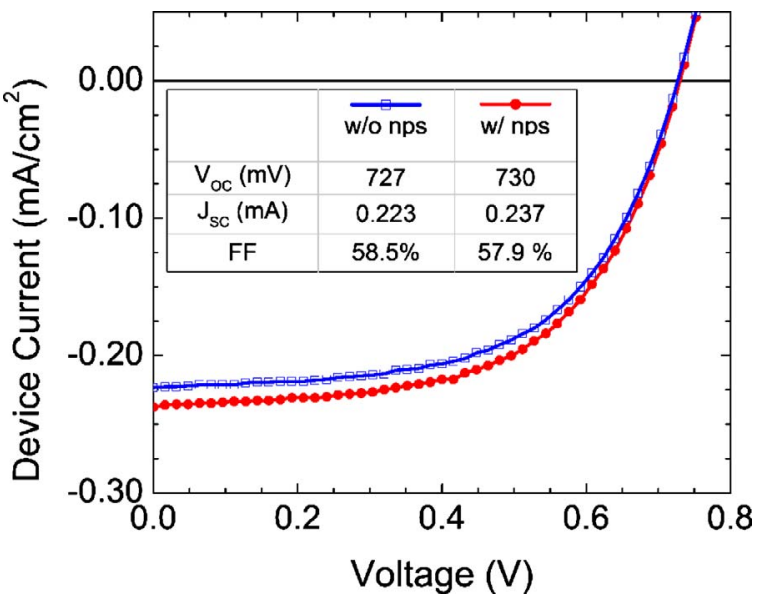

FIG. 3. (Color online) JV measurement of an InGaN QW solar cell, with $200 \mathrm{~nm}$ p-GaN emitter layer, with and without nanoparticles.

tral response measurements for devices before and after nanoparticle deposition using monochromatic $300 \mathrm{~W}$ Xe lamp illumination across the region of the solar spectrum where the cell is absorbing. The cell with a $50 \mathrm{~nm}$ p-GaN emitter layer [Fig. 2(a)] shows a 6\% overall increase in EQE with the addition of nanoparticles. The majority of this enhancement occurs below the GaN band edge, at wavelengths longer than $360 \mathrm{~nm}$, indicating that the photocurrent enhancement is primarily due to the $\mathrm{InGaN}$ QW layer. Figure 2(b) shows the cell with a $200 \mathrm{~nm}$ thick emitter layer. In contrast, the EQE enhancement is predominantly above the $\mathrm{GaN}$ band gap (at wavelengths shorter than $360 \mathrm{~nm}$ ). Here the $\mathrm{EQE}$ increases by $54 \%$ overall due to the nanoparticles. The control cells for both cases underwent the same processing, including both etching and cycling in the thermal evaporator but no change in the photocurrent upon illumination was observed. The notable difference in EQE between the two cells suggests that the light absorption is most strongly enhanced in the region closest to the Ag nanoparticles. The photocurrent enhancement may result from a combination of scattering, local field enhancement, and antireflection coating effects.

Current-voltage measurements of the device with a 200 $\mathrm{nm}$ p-GaN emitter region under the AM 1.5 spectrum at 1 sun illumination $\left(100 \mathrm{~mW} \mathrm{~cm}^{-1}\right)$ are shown in Fig. 3. The presence of nanoparticle arrays on the cells increases the short circuit current appreciably from 0.223 to $0.237 \mathrm{~mA} \mathrm{~cm}^{-2}$. The increase in short circuit current $\left(\mathrm{J}_{\mathrm{sc}}\right)$ is attributed to absorption enhancement evident from the spectral response measurements. The low open circuit voltage for these cells is attributed to damage induced by the dry etch processing required to remove the metal LED contacts. The deterioration in $\mathrm{V}_{\mathrm{oc}}$ that occurred upon etching the cell with $50 \mathrm{~nm} \mathrm{p}-\mathrm{GaN}$ emitter layers made the current-voltage measurements unreliable.

The enhanced $\mathrm{J}_{\mathrm{sc}}$ could also be attributed to an increase in carrier collection efficiency at the surface of the cell. We use one-dimensional device physics simulations (AFORSHet) to model the change in the bandbending and transport for cells with Ag nanoparticle arrays and, specifically, to understand the possible improvement in carrier collection upon nanoparticle layer formation. Other research groups have shown that metal contacts to GaN can cause significant band bending at the semiconductor surface. ${ }^{15}$ Metal layers have 


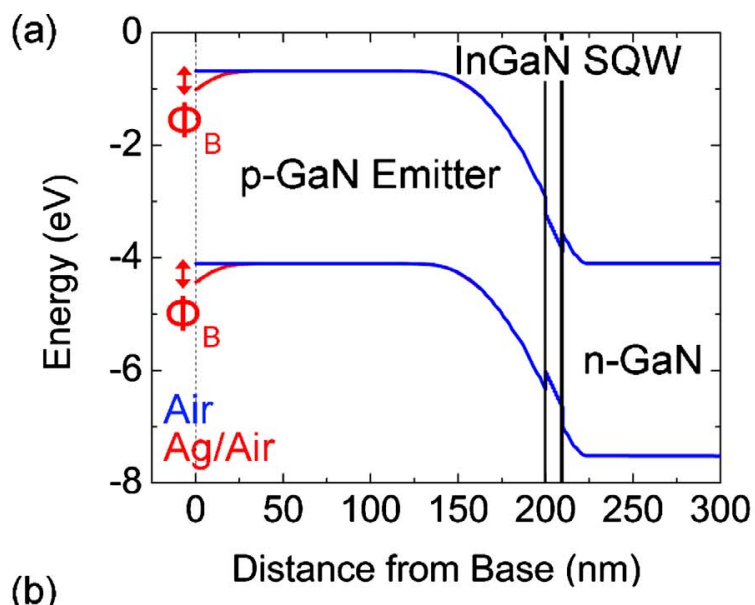

(b)
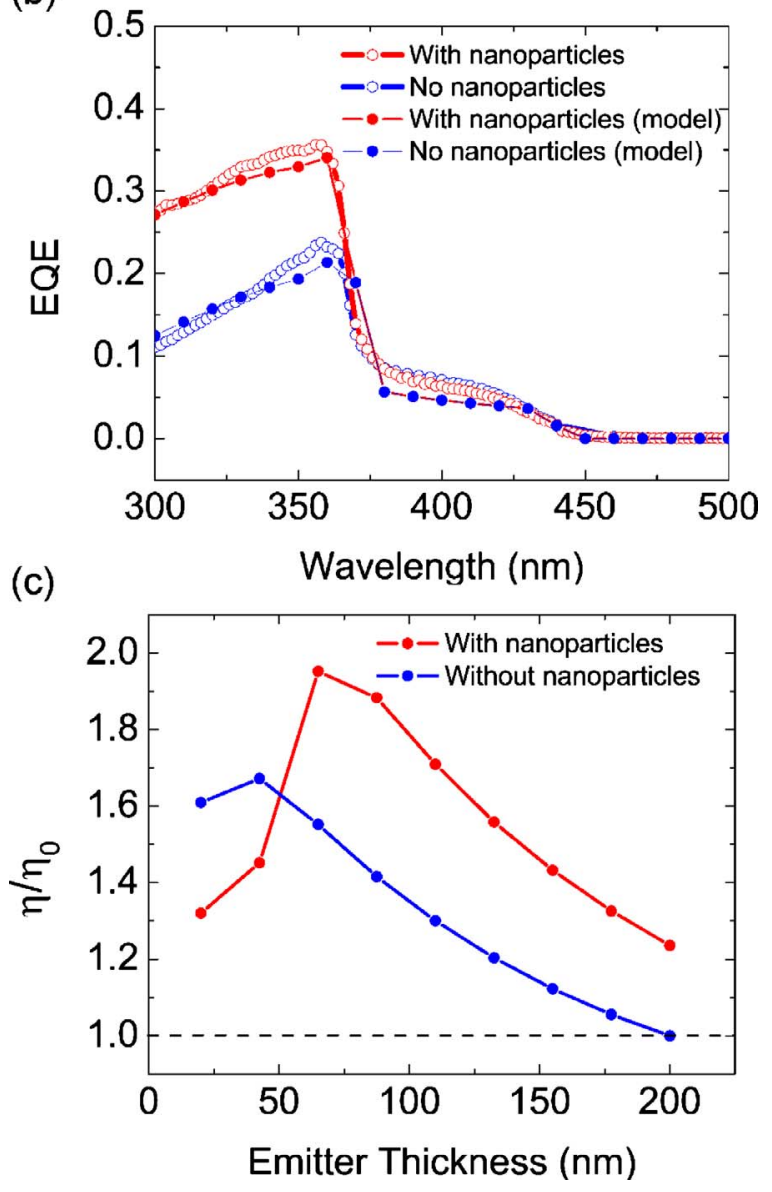

FIG. 4. (Color online) (a) Band-diagram generated in AFORS-Het for cell before and after nanoparticle deposition; (b) AFORS-Het simulation of cell and experimental data before and after nanoparticle deposition; (c) solar cell efficiency simulated for different emitter layer thicknesses.

also been used to improve the contact to both $\mathrm{p}-\mathrm{GaN}$ and $\mathrm{p}-\mathrm{AlGaN}$ layers causing an increase in the depletion width at the surface of the device. ${ }^{16}$ In our model of cells covered with $\mathrm{Ag}$ nanoparticle arrays, we assume band bending equivalent to the $\mathrm{Ag}-\mathrm{GaN}$ barrier height of $0.54 \mathrm{eV}$ at the semiconductor surface [Fig. 4(a)] (Ref. 15) and a reduction of carrier recombination in the p-GaN emitter. The simulated versus the experimental EQE for both control cells and the nanoparticle-decorated $200 \mathrm{~nm}$ p-GaN emitter cell are shown in Fig. 4(b), and show excellent agreement. This EQE calculation assumes a photon flux of $1 \times 10^{16} \mathrm{~cm}^{-2} \mathrm{~s}^{-1}$ and is calculated over the wavelength range from 300 to $500 \mathrm{~nm}$, where the cell is photoactive. After validating the model against the experimental EQE measurements, it was then used as a basis for optimizing device design. We calculated the solar cell efficiency for various emitter thicknesses under the AM 1.5 spectrum at 1 sun illumination and compared these to a $200 \mathrm{~nm}$ thick emitter reference cell without nanoparticles. We found that an optimum emitter layer thickness of $65 \mathrm{~nm}$ doubles the efficiency of the modeled cell [Fig. 4(c)]. We expect that additional improvements could be achieved by thinning the entire cell, so that the semiconductor layers support waveguide modes leading to higher absorption enhancements. However, given the necessity of buffer layer growth and the low dielectric contrast of $\mathrm{GaN}$ to the sapphire substrate, this material system does not lend itself to making thin waveguides.

We have investigated the effects of incorporating arrays of $\mathrm{Ag}$ nanoparticles on $\mathrm{InGaN}$ QW solar cells with emitter layer thickness of 50 and $200 \mathrm{~nm}$. Devices with $50 \mathrm{~nm}$ emitter layers showed an overall $6 \%$ enhancement in photocurrent, with the increase occurring predominantly in the $\mathrm{InGaN}$ active region. Photocurrent was enhanced by $54 \%$ in cells with a $200 \mathrm{~nm}$ thick emitter layer, due primarily to enhanced absorption and collection in the p-GaN emitter and currentvoltage characteristics also improved. Device physics simulations were used to understand the improved device physics of nanoparticle decorated cells and the model developed was used to optimize solar cell design for increased photovoltaic efficiency. The single QW system is a useful platform for the exploration of absorption enhancement from plasmonic scatterers on ultrathin cells designed for optimum photovoltaic response.

This research was supported by the Department of Energy Solar Energy Technology Program, the Global Climate and Energy Program, and the Caltech Center for Sustainable Energy Research. One of the authors (IMP) acknowledges the support of a National Science Foundation Graduate Fellowship.

${ }^{1}$ K. R. Catchpole and A. Polman, Appl. Phys. Lett. 93, 191113 (2008).

${ }^{2}$ S. Pillai, K. R. Catchpole, T. Trupke, and M. A. Green, J. Appl. Phys. 101, 093105(2007).

${ }^{3}$ K. R. Catchpole and S. Pillai, J. Appl. Phys. 100, 044504 (2006).

${ }^{4}$ S. Pillai, K. R. Catchpole, T. Trupke, G. Zhang, J. Zhao, and M. A. Green, Appl. Phys. Lett. 88, 161102 (2006).

${ }^{5}$ D. Derkacs, S. H. Lim, P. Matheu, W. Mar, and E. T. Yu, Appl. Phys. Lett. 89, 093103 (2006).

${ }^{6}$ V. E. Ferry, L. A. Sweatlock, D. Pacifici, and H. A. Atwater, Nano Lett. 8, 4391 (2008).

${ }^{7}$ H. R. Stuart and D. G. Hall, Appl. Phys. Lett. 73, 3815 (1998).

${ }^{8}$ K. Nakayama, K. Tanabe, and H. A. Atwater, Appl. Phys. Lett. 93, 121904 (2008).

${ }^{9}$ P. Campbell and M. A. Green, J. Appl. Phys. 62, 243 (1987).

${ }^{10}$ C. F. Bohren and D. R. Huffman, Absorption and Scattering of Light by Small Particles (Wiley, New York, 1983).

${ }^{11}$ J. Wu, W. Walukiewicz, K. M. Yu, W. Shan, J. W. Ager, E. E. Haller, H. Lu, W. J. Schaff, W. K. Metzger, and S. Kurtz, J. Appl. Phys. 94, 6477 (2003).

${ }^{12}$ X. Chen, K. Matthews, D. Hao, W. Schaff, and L. Eastman, Phys. Status Solidi A 205, 1103 (2008).

${ }^{13}$ O. Jani, I. Ferguson, C. Honsberg, and S. Kurtz, Appl. Phys. Lett. 91, 132117 (2007).

${ }^{14}$ R. H. Horng, S. T. Lin, Y. L. Tsai, M. T. Chu, W. Y. Liao, M. H. Wu, R. M. Lin, and Y. C. Lu, IEEE Electron Device Lett. 30, 724 (2009).

${ }^{15}$ A. C. Schmitz, A. T. Ping, M. A. Khan, Q. Chen, J. W. Yang, and I. Adesida, J. Electron. Mater. 27, 255 (1998).

${ }^{16}$ Y. J. Lin, Jpn. J. Appl. Phys., Part 2 45, L86 (2006). 